\begin{tabular}{c} 
Brazilian Journal \\
of Chemical \\
Engineering \\
\hline
\end{tabular}

ISSN 0104-6632

Printed in Brazil

www.abeq.org.br/bjche

Vol. 31, No. 02, pp. 347 - 354, April - June, 2014

dx.doi.org/10.1590/0104-6632.20140312s00002587

\title{
MODELING THE MICROBIAL GROWTH OF TWO Escherichia coli STRAINS IN A MULTI-SUBSTRATE ENVIRONMENT
}

\author{
M. E. Poccia ${ }^{1}$, A. J. Beccaria ${ }^{1}$ and R. G. Dondo ${ }^{2 *}$ \\ ${ }^{1}$ Laboratorio de Fermentaciones, Facultad de Bioquímica y Ciencias Biológicas, \\ Universidad Nacional del Litoral Ciudad Universitaria, Paraje El Pozo (S3000ZAA), Santa Fe, Argentina. \\ ${ }^{2}$ Instituto de Desarrollo Tecnológico para la Industria Química, INTEC, CONICET, Phone: + 5442559174 77, \\ Fax: + 54 42532965, Universidad Nacional del Litoral, Güemes 3450, (3000) Santa Fe, Argentina. \\ "E-mail: rdondo@santafe-conicet.gov.ar
}

(Submitted: March 6, 2013 ; Revised: August 1, 2013 ; Accepted: September 10, 2013)

\begin{abstract}
The microbial growth in multi-substrate environments may be viewed as an optimal resources allocation problem. The optimization aims at maximizing some biological objective like the biomass growth. The models developed using this hypothesis are called "cybernetic" and they represent the complex cell structure as an optimizing function that regulates the intracellular enzymatic machinery. In this work, a cybernetic model was developed to represent the growth of two E. coli strains (JM 109 and BL 21 -DE3-) on a medium containing glucose and glycerol as carbon and energy sources. The model was able to accurately simulate the biomass growth, the substrates consumption and the growth-rate profiles.

Keywords: Cybernetic model; Optimal control; Escherichia coli; Multi-substrates; Glycerol.
\end{abstract}

\section{INTRODUCTION}

Modeling the kinetics of a fermentation process in complex media remains a challenge for biochemical engineers and biotechnologists. Although empirical models such as the Monod equation describe the growth kinetics on a single substrate, they are usually not useful for a multi-substrate environment. Different growth phenomena such as sequential utilization of substrates, simultaneous substrates consumption and co-metabolism of substrates may be observed in these environments (Bajpai-Dikshit et al., 2003). There have been numerous attempts to model these phenomena with an unified representation. A successful approach to these phenomena was the cybernetic modeling concept developed by Ramkrishna and co-workers (Kompala et al., 1984; Kompala et al., 1986; Varner and Ramkrishna, 1999; Song and Ramkrishna, 2010).

In biochemical systems, many reactions are cata- lyzed by enzymes, which may be inhibited or activated by regulatory effects on the genes that code for these enzyme activities. As a result, the changes at the regulatory level may impact the dynamics of the biochemical pathways. Cybernetic models expand the traditional ordinary differential equations (ODE) models by incorporating control laws that manipulate the enzyme synthesis and/or activity in order to drive the pathways toward a stated biological goal. The framework is based on the postulate that biological systems operate according to an optimal resources allocation objective. This objective is developed as a result of evolutionary pressures imposed on the organism. Various criteria have been proposed as the optimality criterion for the allocation of critical resources: maximum biomass productivity (Kompala et al., 1984), maximization of the final biomass concentration (Dhurjati et al., 1985) and matching-law allocation (Kompala et al., 1986). The cybernetic modeling concept hypothesizes that the

*To whom correspondence should be addressed 
cellular objectives of the wild-type and the genetically altered systems are identical (Varner and Ramkrishna, 1998), supporting the use of the same objective function for predicting changes in dynamics due to regulatory effects.

Escherichia coli (E. coli) is perhaps the most studied bacterium. It can adapt itself to very different growth conditions. High density cultures of several strains of E. coli are currently used to produce recombinant proteins due to its high volumetric productivity; see e.g. Shiloach J. and Fass R. (2005) and Huang et al. (2012). This, in practice, has transformed $E$. coli into a "factory" of recombinant proteins and many pharmaceutical products are produced in this way.

E. coli is capable of consuming either glucose or glycerol as carbon and energy $(\mathrm{C} / \mathrm{E})$ sources. Also it may consume either organic nitrogen $(\mathrm{N})$ from sources such as peptones or inorganic $(\mathrm{N})$-salts. It is, therefore, extremely versatile. Although glucose is the classical (C/E)-source, glycerol is an attractive alternative because it has become an inexpensive and abundant $(\mathrm{C} / \mathrm{E})$-source due to its generation as an inevitable by-product of biodiesel (Yazdani and Gonzalez, 2007; Gonzalez et al., 2008) and its overproduction has become an issue of concern (Albarelli et al., 2011). The replacement of glucose by glycerol as $(\mathrm{C} / \mathrm{E})$-source also allows obtaining higher biomass densities, probably because of a higher solubility and a slower catabolism, which avoids acetate production (Kortz et al., 1995). This makes glycerol a cheap option as the main substrate for numerous industrial cultures. So, it is desirable to adjust the E. coli growth conditions to promote glycerol consumption rather than glucose consumption. Nevertheless, glucose may be useful as a growth initiator. Consequently, in this work a cybernetic model has been used to describe the dynamics of glucose and glycerol consumption by two E. coli strains (JM 109 and BL $21-$ DE3-) in batch and batch + substrate-injection cultures.

\section{MATERIALS AND METHODS}

\section{Microorganisms and Medium}

E. coli BL 21 (DE3) and JM 109 strains were grown on MEP medium adjusted according to guidelines proposed by Giordano et al. (2010): acid hydrolysate of casein -AHC- $(30 \mathrm{~g} / \mathrm{L})$; yeast extract $(27.5 \mathrm{~g} / \mathrm{L}) ; \mathrm{NaCl}(1 \mathrm{~g} / \mathrm{L}) ;$ glycerol $(1 \mathrm{~g} / \mathrm{L})$ and glucose $(1 \mathrm{~g} / \mathrm{L})$. Sterilization was done at $121{ }^{\circ} \mathrm{C}$ for $15 \mathrm{~min}$. AHC and yeast extract were acquired from Britania, Argentina. $\mathrm{NaCl}$, glycerol and glucose were acquired from Cicarelli Laboratorios, Argentina. Although the concentrations of AHC, yeast extract and $\mathrm{NaCl}$ are relatively high, the increase in the yield with respect to produced biomass overcomes this disadvantage (Giordano et al., 2010).

\section{Inoculum Preparations and Culture Conditions}

A loopful of each strain (stored in MEP slant agar at $4{ }^{\circ} \mathrm{C}$ ) was inoculated into a $250 \mathrm{ml}$ Erlenmeyer flask containing $30 \mathrm{ml}$ of sterilized MEP medium and grown overnight on a rotary shaker at $200 \mathrm{RPM}$ and $30^{\circ} \mathrm{C}$. Aliquots of these cultures were pipetted into $250 \mathrm{ml}$ Erlenmeyer flasks containing $30 \mathrm{ml}$ of the same medium to obtain an initial optical density at $600 \mathrm{~nm}$ (OD600) of 0.2. The cultures were incubated at $200 \mathrm{rpm}$ at $30{ }^{\circ} \mathrm{C}$. Several samples were withdrawn from each culture.

\section{Samples Analysis}

OD600 was measured and used as an estimate of the cell mass $(1 \mathrm{OD}=0.20 \mathrm{~g}$ dry weight $/ \mathrm{L})$. Aliquots were centrifuged $(10.000 \times \mathrm{g}, 5$ minutes $)$ and supernatants were stored at $-20^{\circ} \mathrm{C}$ for further analysis.

\section{Analytical}

Glucose and glycerol concentrations were determined in thawed samples. The enzymatic colorimetric assays from Glicemia Enzimática AA kit and TG Color GPO/PAP AA kit were employed for glucose and glycerol quantification, respectively. Both were purchased from Wiener Lab, Argentina. Determination accuracy is $90.7 \mathrm{mg} / \mathrm{dL} \pm 2.20 \mathrm{mg} / \mathrm{dL}$ (C.V. $=1.39 \%$ ) for glucose concentration and $1.14 \mathrm{~g} / \mathrm{L} \pm 0.021 \mathrm{~g} / \mathrm{L}$ (C.V. $=1.82 \%)$ for the glycerol concentration.

\section{Computational Tools}

ODE and differential-algebraic equations (DAE) were solved by using the MATLAB ODE 45 solver (Mathworks, Natick, MA, USA) and the optimal control problems were solved by the GPOPS 4.1 toolbox (Rao et al, 2011) running on MATLAB 7.6.

\section{MODEL DEVELOPMENT}

The microbial growth is represented here in a way similar to the cybernetic model developed by Kompala et al. (1984):

$$
X+Y_{X / S_{i}} S_{i} \stackrel{e_{i}}{\longrightarrow}\left(1+Y_{X / S_{i}}\right) X
$$


In the assimilation of the $i^{\text {th }}$ substrate by the biomass $X$, it is assumed that there is one lumped keyenzyme which plays the bottleneck role and hence must be synthesized before the substrate is utilized for growth. The synthesis of the $i^{\text {th }}$ key enzyme in the presence of the $i^{\text {th }}$ substrate can be represented as follows:

$$
X \stackrel{S_{i}}{\longrightarrow} X+e_{i}
$$

The symbols $X, S_{i}$ and $e_{i}$ were used to represent the pseudo-reaction "stoichiometry", but subsequently $X$ will represent the biomass concentration, $S_{i}$ will represent the concentration of the $i^{\text {th }}$ substrate and $e_{i}$ will represent the normalized intracellular level of the enzyme necessary to consume the $i^{\text {th }}$ substrate.

The actual specific growth $\mu$ will arise from the sequential/simultaneous growth on all substrates. According to the modified Monod's expression (Kompala et al., 1984), $\mu$ will be:

$\mu=\sum_{i} \mu_{i}^{\max } e_{i} \frac{S_{i}}{k_{i}+S_{i}}$

where $i$ is the substrate indicator, $\mu_{i}^{\max }$ is the maximum specific growth rate on the substrate $i$ and $k_{i}$ is the Monod substrate saturation constant. The ODE for the substrate consumption and the specific enzyme formation rate in a batch environment are given by:

$$
\begin{aligned}
\frac{d S_{i}}{d t} & =-\frac{1}{Y_{X / S i}} \mu_{i}^{\max } e_{i} \frac{S_{i}}{k_{i}+S_{i}} X \\
\frac{d e_{i}}{d t} & =a_{i} u_{i}-b_{i} e_{i}
\end{aligned}
$$

Some variations in the shape of the above equation were proposed (Kompala et al., 1984 and 1986; Dhurjati et al., 1985; Doshi et al., 1997), but the fundamental structure of the ODE system remains unaltered. To model the E. coli-strain growth on a medium with glucose and glycerol, we selected the matching-law allocation model (Kompala et al., 1986) and the whole model-system may be written as:

$$
\frac{d X}{d t}=\sum_{i} \mu_{i}^{\max } \frac{S_{i}}{k_{i}+S_{i}} e_{i} v_{i} X
$$

$$
\begin{aligned}
& \frac{d S_{i}}{d t}=-\frac{1}{Y_{X / S i}} \mu_{i}^{\max } \frac{S_{i}}{k_{i}+S_{i}} e_{i} v_{i} X \\
& \frac{d e_{i}}{d t}=\alpha_{i} \frac{S_{i}}{k_{i}^{\prime}+S_{i}} u_{i}-\beta_{i} e_{i}
\end{aligned}
$$

The cybernetic variables $u_{i}$ and $v_{i}$ are computed from the following algebraic equations:

$$
\begin{gathered}
u_{i}=\frac{\mu_{i}^{\max } \frac{S_{i}}{k_{i}+S_{i}} e_{i}}{\sum_{j}\left(\mu_{j}^{\max } \frac{S_{j}}{k_{j}+S_{j}} e_{j}\right)} \\
v_{i}=\frac{\mu_{i}^{\max } \frac{S_{i}}{k_{i}+S_{i}} e_{i}}{\max _{j}\left(\mu_{j}^{\max } \frac{S_{j}}{k_{j}+S_{j}} e_{j}\right)}
\end{gathered}
$$

The parameters $\alpha_{i}$ and $\beta_{i}$ stand respectively for the specific activation rate and degradation rate of the key enzyme $e_{i}$. The right-hand-side of Eq. (8) neglects a term of intracellular dilution of the enzymelevel due to the biomass growth that is presented in the model by Kompala et al. (1986). Nevertheless, this dilution effect can be lumped into the $\beta_{i}$ parameter and the DAE will not lose predictive capability at all. The maximum specific growth rates $\mu_{i}^{\max }$ and the saturation constants $k_{i}$ are usually estimated by fitting data from the exponential phases corresponding to the respective substrates. Also it is usually assumed that $k_{i}=k_{i}^{\prime}$.

\section{RESULTS AND DISCUSSION}

Experimental results for E. coli strains growing in a medium with glucose and glycerol are summarized in Table A.1 of the appendix. In both cultures we observed three growth stages: a fast growth stage on glucose followed by a slower growth stage on glycerol and a very slow growth phase utilizing the peptides from $\mathrm{AHC}$ as $(\mathrm{C} / \mathrm{E})$-source. Hence, the whole culture seems to be triauxic. The model-parameters obtained by minimizing the least square errors between the experimental data and the model predictions are reported in Table 1. To avoid an over-parametrization of the model, the values of parameters $\alpha_{i}$ and $\beta_{i}$ were prefixed. So, growth parameters in the late culture phase, without glucose and glycerol, 
were forced to be identical in both least-squares fittings. Figures 1 and 2 compare the experimental data with the model predictions.

Table 1: Model parameters for the least squares fitting of experimental data from both batch runs.

\begin{tabular}{|l|c|c|c|c|c|}
\hline $\begin{array}{l}\boldsymbol{i} \text { C/E- } \\
\text { source }\end{array}$ & $\boldsymbol{\mu}_{\boldsymbol{i}}{ }^{\text {max }}$ & $\boldsymbol{k}_{\boldsymbol{i}}$ & $\boldsymbol{Y}_{X / S i}$ & $\boldsymbol{\alpha}_{\boldsymbol{i}}{ }^{{ }^{*}}$ & $\boldsymbol{\beta}_{\mathrm{i}}{ }^{{ }^{*}}$ \\
\hline \multicolumn{6}{|c|}{ E. coli JM 109 strain } \\
\hline Glucose & 0.53 & 0.01 & 0.23 & 1 & 1 \\
Glycerol & 0.46 & 0.35 & 0.38 & 1 & 1 \\
AHC & 0.04 & 0.01 & 0.21 & 1 & 1 \\
\hline \multicolumn{6}{|c|}{ E. coli BL 21 (DE3) strain } \\
\hline Glucose & 0.76 & 0.01 & 0.26 & 1 & 1 \\
Glycerol & 0.54 & 0.65 & 0.25 & 1 & 1 \\
AHC & 0.04 & 0.01 & 0.23 & 1 & 1 \\
\hline
\end{tabular}

*Prefixed to avoid overparametrization

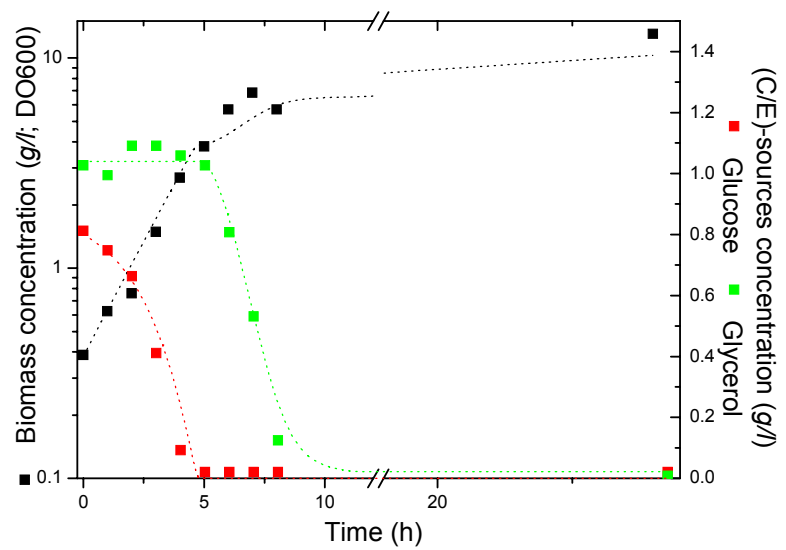

Figure 1: Model predictions (dotted lines) vs. experimental data (square symbols) for the E. coli JM 109 strain.

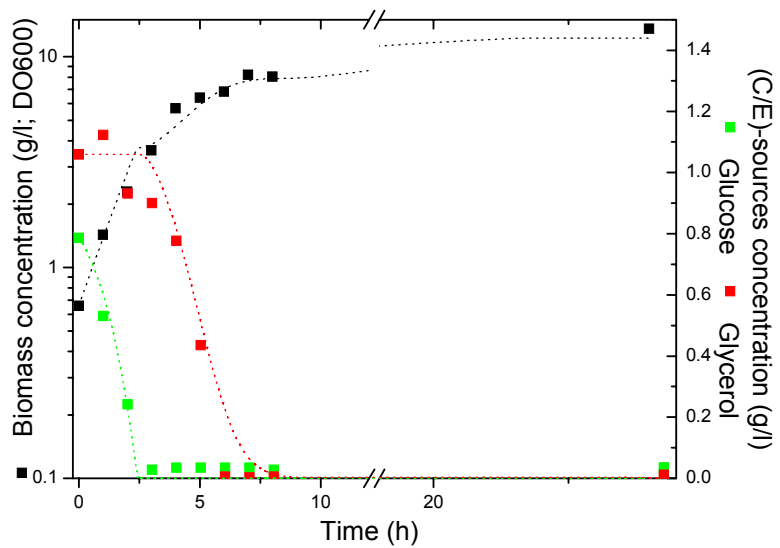

Figure 2: Model predictions (dotted lines) vs. experimental data (square symbols) for the E. coli $\mathrm{BL}$ 21 (DE3) strain.

Since experimental data for the growth on peptides from AHC is scarce, the parameters for this stage present a significant degree of uncertainty.
Nevertheless, this should not affect the design of a medium with glucose and glycerol as the main $(\mathrm{C} / \mathrm{E})$ sources because the growth on peptides from AHC is much slower than the growth on the former substrates. As our aim is to promote the utilization of glycerol while using glucose just as a growth-initiator, to design a batch + injection culture, we solved an optimal control problem. In that problem, the glycerol concentration may be changed by glycerol injections up to the final culture-time of $t_{f}=10 \mathrm{~h}$. The growth stage on peptides from AHC was neglected in the optimization and the problem can be stated as follows:

Maximize $X\left(t_{f}\right) V\left(t_{f}\right)$

Subject to:

$\frac{d V}{d t}=F$

$\frac{d X}{d t}=\sum_{i} \mu_{i}^{\max } \frac{S_{i}}{k_{i}+S_{i}} e_{i} v_{i} X-F \frac{X}{V}$

$\frac{d S_{i}}{d t}=-\frac{1}{Y_{X / S i}} \mu_{i}^{\max } \frac{S_{i}}{k_{i}+S_{i}} e_{i} v_{i} X+\frac{S_{i}^{F}}{V} F-F \frac{S_{i}}{V}$

$\frac{d e_{i}}{d t}=\alpha_{i} \frac{S_{i}}{k_{i}^{\prime}+S_{i}} u_{i}-\beta_{i} e_{i}$

$\frac{d Q_{2}}{d t}=F S_{2}^{F}$

Here $F$ is the manipulated variable and the states are the culture volume $(V)$, the biomass concentration $(X)$, the glucose concentration $\left(S_{1}\right)$, the glycerol concentration $\left(S_{2}\right)$, the normalized intracellular level of enzymes $\left(e_{1}\right.$ and $\left.e_{2}\right)$ and the quantity $Q_{2}$ of injected glycerol. Since the injection is glucose-free $S_{1}{ }^{F}=0 \mathrm{~g} / \mathrm{L}$ and $Q_{1}=0 \mathrm{~g}$. The concentration of glycerol in the feed is $S_{2}^{F}=5 \mathrm{~g} / \mathrm{L}$. The objective (Eq. 11) is the maximization, at the final run-time $t_{f}=10 \mathrm{~h}$, of the biomass quantity subject to the constraints (Eqs. (12)-(16)) of a fed-batch bioreactor. The cybernetic variables $u_{i}$ and $v_{i}$ are computed from Eqs. (9) and (10). In addition, the constraint (17) imposes an upper bound $Q_{2}{ }^{\mathrm{max}}$ to the quantity of injected glycerol:

$Q_{2} \leq Q_{2}{ }^{\max }$ 
The optimal control problem is defined by the objective function (Eq. 11), the differential equations (Eqs. (12)-(16)), the algebraic Equations (9)-(10) and the state constraint (Eq. 17). It was coded on GPOPS 4.1 (Rao et al., 2011) and solved in a $2.0 \mathrm{GHz} 16$ GRAM PC.

We solved several instances of this optimal control problem by varying the initial states and the kinetic parameter values. In all cases, the glycerol addition consisted of an injection just before the start of the glycerol consumption. The explanation of this feed-shape is fairly simple. Since $\mu_{1}{ }^{\max }$ for glucose is larger than for other substrates and the glucosesaturation constant $k_{1}$ is also very low, the glucose is preferred in the sequential substrate utilization. Because $\mu_{1}{ }^{\max }>\mu_{2}{ }^{\max }$ and $k_{1}<k_{2}$, the optimal profile will always yield a sequential substrates utilization.

So, an early injection of the "slow" substrate will not increase the actual growth rate at all and it might even brake the activation of the enzyme necessary for the degradation of the "fast" substrate (i.e., if $\mu_{1}{ }^{\max }$ is slightly larger than $\mu_{2}{ }^{\max }$ ). Hence, the injection will start just after the glucose depletion and will occur as early as possible. The goal is to minimize the effect of the Monod saturation-term for glycerol, aiming at:

$\mu_{2}^{\max } \frac{S_{2}}{k_{2}+S_{2}} e_{2} v_{2} \approx \mu_{2}^{\max }$

An optimal substrate-addition profile is illustrated in Figure 3. The kinetic parameters and initial states of such a profile are reported in Table 2. The kinetic and yield parameters are those that fit the batch culture of the E. coli BL 21 (DE3) strain.

After this numerical experiment, we performed a batch + injection culture to grow the E. coli BL 21 (DE3) strain under the environmental conditions computed from the rule derived from the above optimal control problem. That rule consists of injecting glycerol just after glucose exhaustion.

Table 2: Model parameters and initial states for the optimal control problem solved to design the glycerol injection profile.

\begin{tabular}{|c|c|c|c|c|c|c|}
\hline \multicolumn{3}{|c|}{ Time horizon } & & \multicolumn{3}{|c|}{$t_{f}=10 \mathrm{~h}$} \\
\hline \multicolumn{7}{|c|}{ Initial states } \\
\hline$X(0)$ & $S_{1}(0)$ & $S_{2}(0)$ & $e_{1}(0)$ & $e_{2}(\mathbf{0})$ & $V(0)$ & $Q(0)$ \\
\hline 0.2 & 2.6 & 1.2 & 0.9 & 0.1 & 1.0 & 0 \\
\hline \multicolumn{7}{|c|}{ Yield and kinetic parameters } \\
\hline$i$ C-source & $\mu_{i}^{\mathrm{m}}$ & & & $Y_{X / S i}$ & $\boldsymbol{\alpha}_{i}$ & $\boldsymbol{\beta}_{i}$ \\
\hline Glucose & 0.7 & & & 0.26 & 1 & 1 \\
\hline Glycerol & 0.5 & & & 0.25 & 1 & 1 \\
\hline
\end{tabular}
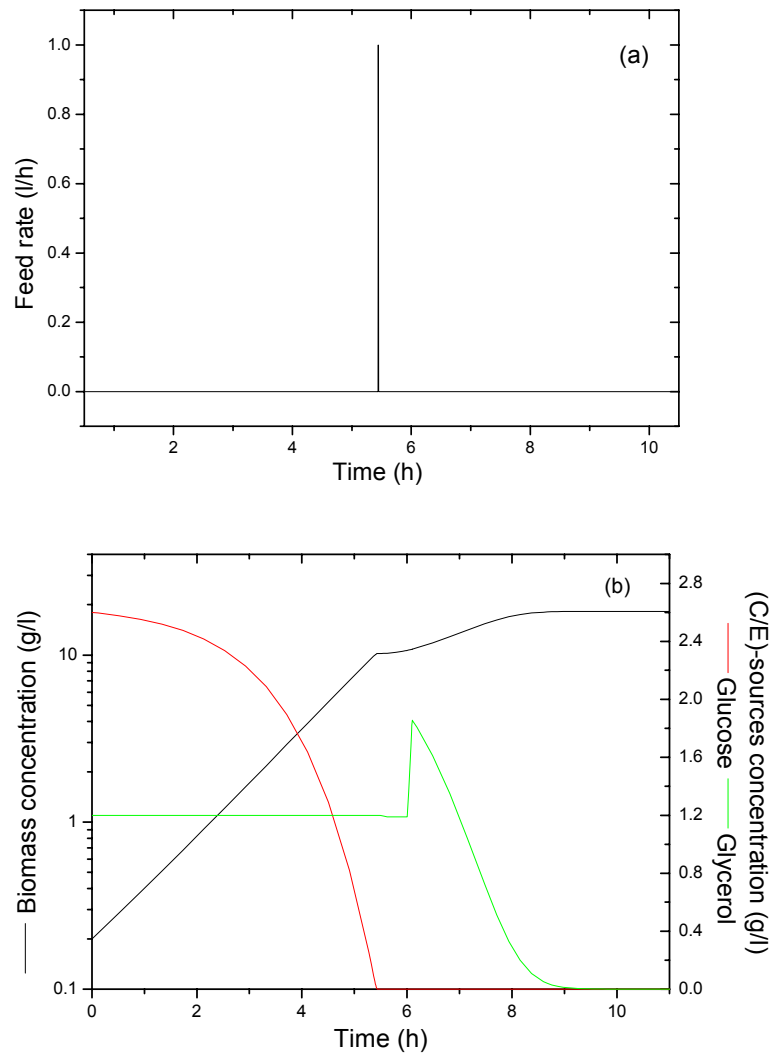

Figure 3: An optimal glycerol injection profile (a) and the evolution of the biomass and substrates concentrations (b).

The experimental results for such a run are summarized in Table A2 of the Appendix. The least squares fit of the model to the data yielded the parameters reported in Table 3 and Figure 4 compares the model predictions with the experimental data.

The experimental results indicate a growth rate on glucose similar to the growth rate observed in the batch culture, but also indicate a simultaneous consumption of glucose and glycerol after $t=3 \mathrm{~h}$ and up to the time of the glycerol-injection. The simultaneous substrates-consumption was not caused

Table 3: Model parameters for the least squares fitting of the experimental results presented in Table A2.

\begin{tabular}{|c|c|c|c|c|c|}
\hline$i \mathrm{C}$-source & $\mu_{i}^{\max }$ & $\boldsymbol{k}_{i}$ & $Y_{X / S i}$ & $\boldsymbol{\alpha}_{i}{ }^{*}$ & $\boldsymbol{\beta}_{i}{ }^{*}$ \\
\hline \multicolumn{6}{|c|}{ E. coli BL 21 (DE3) strain } \\
\hline Glucose & 0.75 & 0.12 & 0.66 & 1 & 1 \\
\hline Glycerol & 0.14 & 0.60 & 0.25 & 1 & 1 \\
\hline \multicolumn{3}{|c|}{\begin{tabular}{|l} 
Injection time \\
Injected volume \\
$S_{2}{ }^{F}$
\end{tabular}} & \multicolumn{3}{|c|}{$\begin{array}{r}6 \mathrm{~h} \\
0.1 \mathrm{ml} / \mathrm{ml} \text { culture } \\
5 \mathrm{~g} / \mathrm{l} \\
\end{array}$} \\
\hline
\end{tabular}




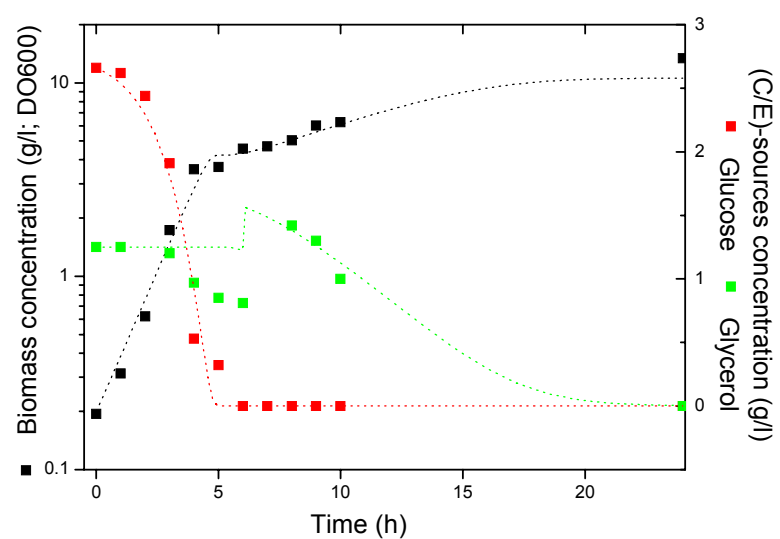

Figure 4: Model predictions (dotted lines) vs. experimental (square symbols) data for the batch + injection culture with the $E$. coli BL 21 (DE3) strain.

by the injection because it occurred before. Before this unexpected behavior and after the glycerol injection, the agreement is quite good. Therefore, the remaining experimental data seems to validate the model also in a batch + injection mode.

\section{CONCLUSIONS}

A cybernetic model based on the maximization of the instantaneous specific growth rate hypothesis is able to represent a variety of experimental growth data involving diauxic and triauxic growth. The model utilizes a lumped representation of the cell populations, and the microbial growth on various $(\mathrm{C} / \mathrm{E})$-sources can be treated as a multivariable constrained optimization problem, as proposed by Kompala et al (1986). This model was chosen to predict the growth of two E. coli strains on a medium with glucose and glycerol. It can accurately represent the growth dynamics of both strains in batch runs.

To design a batch + injection culture, an optimal control problem was subsequently solved. As a consequence, an injection rule was derived and an additional experiment was performed. In that case, the agreement between the model predictions and the experimental data was not as good as for batch cultures, although this cannot be attributed to the glycerol injection. So, the empirical rule remains valid.

\section{ACKNOWLEDGEMENTS}

This work was supported by grants from Universidad Nacional del Litoral (CAI+D 2009-335 and CAI+D 2010 12-0035). We also want to thank the reviewer for helpful observations.

\section{NOMENCLATURE}

$a_{i} \quad$ Specific activation rate for the $i^{\text {th }}$ enzyme

$b_{i} \quad$ Specific inactivation rate for the $i^{\text {th }}$ enzyme

$e_{i} \quad$ Normalized intracellular level of the $i^{\text {th }}$ enzyme

$k_{i} ; k_{i}^{\prime} \quad$ Monod substrate saturation $\mathrm{g} / \mathrm{L}$ constant

$F \quad$ Feed rate

$Q_{i} \quad$ Mass of the $i^{\text {th }}$ substrate injected to the culture

$S_{i} \quad$ Concentration of the $i^{\text {th }} \quad \mathrm{g} / \mathrm{L}$

$S_{i}^{F} \quad$ Concentration of the $i^{t h}$ substrate in the feed

$u_{i} \quad$ Cybernetic variable for controlling the synthesis of the $i^{\text {th }}$ enzyme

$v_{i} \quad$ Cybernetic variable for regulating the activity of the $i^{\text {th }}$ enzyme

$V \quad$ Culture volume

$X \quad$ Biomass concentration

$Y_{X / S i} \quad$ Yield of biomass on the $i^{\text {th }}$ $\mathrm{g} / \mathrm{L}$ substrate

\section{Greek Symbols}

$\alpha_{i} \quad$ Specific activation rate of the $i^{\text {th }}$ enzyme

$\beta_{i} \quad$ Specific inactivation rate of

the $i^{\text {th }}$ enzyme $e_{i}$

$\mu \quad$ Actual specific growth rate $\quad \mathrm{h}^{-1}$

$\mu_{i}^{\max } \quad$ Maximum growth rate on $\quad \mathrm{h}^{-1}$

\section{REFERENCES}

Albarelli, J., Santos, D. and Holanda, M., Energetic and economic evaluation of waste glycerol cogeneration in Brazil. Brazilian Journal of Chemical Engineering, 28(4), 691-698 (2011).

Bajpai-Dikshit, J., Suresh, A. and Venkatesh, K., An optimal model for representing the kinetics of growth and product formation by Lactobacillus rhamnosus on multiple substrates. Journal of Bioscience and Bioengineering, 96(5), 481-486 (2003). 
Dhurjati, P., Ramkrishna, D., Flickinger, M. C. and Tsao, G. T., A cybernetic view of microbial growth: Modeling of microbes as optimal strategists. Biotechnology and Bioengineering, 27, 1-9 (1985).

Doshi, P., Rengaswamy, R. and Venkatesh, K., Modelling of microbial growth for sequential utilization in a multisubstrate environment. Process Biochemistry, 32(8), 643-650 (1997).

Giordano, P., Martínez, H., Iglesias, A., Beccaria, A. and Goicoechea, H., Application of response surface methodology and artificial neural networks for optimization of recombinant Oryza sativa non-symbiotic haemoglobin-1 production by Escherichia coli in medium containing byproduct glycerol. Bioresource Technology, 101, 7537-7544 (2010).

Gonzalez, R., Murarka, A., Dharmadi, Y. and Yazdani, S., A new model for the anaerobic fermentation of glycerol in enteric bacteria: Trunk and auxiliary pathways in Escherichia coli. Metabolic Engineering, 10, 234-245 (2008).

Huang, C-Jr, Lin, H., Yang, X., Industrial production of recombinant therapeutics in Escherichia coli and its recent advancements. Journal of Industrial Microbiology and Biotechnology, 39, 383-399 (2012).

Kompala, D., Ramkrishna, D., Jansen, N. and Tsao, G., Investigation of bacterial growth on mixed substrates: Experimental evaluation of cybernetic models. Biotechnology and Bioengineering, 28, 1044-1055 (1986).
Kompala, D., Ramkrishna, D. and Tsao, G., Cybernetic modeling of microbial growth on multiple substrates. Biotechnology and Bioengineering, 27, 1272-1281 (1984).

Kortz, D., Rinas, U., Hellmuth, K., Sanders, E. and Deckwer, W., Simple fed-batch technique for high cell density cultivation of Escherichia coli. Journal of Biotechnology, 39(1) 59-65 (1995).

Rao, A. V., Benson, D., Darby, C., Francolin, C., Patterson, M., Sanders, I. and Huntington, G. T., User's Manual for GPOPS Version 4.0 (2011).

Shiloach, J., Fass, R., Growing E. coli to high cell density. A historical perspective on method development. Biotechnology Advances, 23(5), 345357 (2005).

Song, H. S. and Ramkrishna, D., Prediction of metabolic function from limited data: Lumped Hybrid Cybernetic Modeling (L-HCM). Biotechnology and Bioengineering, 106(2), 271-284 (2010).

Varner, J. and Ramkrishna, D., Metabolic engineering from a cybernetic perspective. 1. Theoretical preliminaries. Biotechnology Progress, 15(3), 407425 (1999).

Varner, J. and Ramkrishna, D., Application of cybernetic models to metabolic engineering: Investigation of storage pathways. Biotechnology and Bioengineering, 58(2-3), 282-291 (1998).

Yazdani, S. and Gonzalez, R., Anaerobic fermentation of glycerol: a path to economic viability for the biofuels industry. Current Opinions in Biotechnology, 18, 213-219 (2007). 


\section{APPENDIX}

Table A.1: Experimental growth data for batch culture of $E$. coli strains JM 109 and BL 21 (DE3) on a mixed medium of glucose and glycerol.

\begin{tabular}{|c|c|c|c|}
\hline \multicolumn{1}{|c|}{ Time (h) } & Biomass (g/l) & Glucose (g/l) & Glycerol (g/l) \\
\hline \multicolumn{4}{|c|}{ E. coli JM 109 strain } \\
\hline 0 & 0.39 & 0.81 & 1.03 \\
1 & 0.62 & 0.75 & 0.99 \\
2 & 0.76 & 0.66 & 1.09 \\
3 & 1.49 & 0.41 & 1.09 \\
4 & 2.70 & 0.09 & 1.06 \\
5 & 3.80 & 0.02 & 1.03 \\
6 & 5.70 & 0.02 & 0.81 \\
7 & 6.85 & 0.02 & 0.53 \\
8 & 5.70 & 0.02 & 0.12 \\
24 & 13.06 & 0.02 & 0.01 \\
\hline \multicolumn{5}{|c|}{ E. coli BL 21 (DE3) strain } \\
1 & 0.66 & 0.79 & 1.06 \\
2 & 1.43 & 0.53 & 1.12 \\
3 & 2.29 & 0.24 & 0.93 \\
4 & 3.60 & 0.03 & 0.90 \\
5 & 5.70 & 0.03 & 0.78 \\
6 & 6.39 & 0.03 & 0.44 \\
7 & 6.85 & 0.03 & 0.01 \\
8 & 8.22 & 0.03 & 0.01 \\
24 & 8.05 & 0.03 & 0.01 \\
& 13.60 & 0.03 & 0.01 \\
\hline
\end{tabular}

Table A.2: Experimental growth data for batch + injection culture of $E$. coli BL 21 (DE3) strain on a mixed medium of glucose and glycerol.

\begin{tabular}{|c|c|c|c|}
\hline Time (h) & Biomass (g/l) & Glucose (g/l) & Glycerol (g/l) \\
\hline \multicolumn{3}{|c|}{ E. coli BL 21 (DE3) } \\
\hline 0 & 0.19 & 2.66 & 1.25 \\
1 & 0.31 & 2.62 & 1.25 \\
2 & 0.62 & 2.44 & NA \\
3 & 1.73 & 1.91 & 1.20 \\
4 & 3.57 & 0.53 & 0.97 \\
5 & 3.67 & 0.32 & 0.85 \\
6 & 4.56 & 0.00 & 0.81 \\
7 & 4.69 & 0.00 & NA \\
8 & 5.04 & 0.00 & 1.42 \\
9 & 6.02 & 0.00 & 1.30 \\
10 & 6.74 & 0.00 & 1.00 \\
24 & 13.4 & 0.00 & 0.00 \\
\hline
\end{tabular}

